\title{
Deleterious PLCB4 Gene Mutation
}

National Cancer Institute

\section{Source}

National Cancer Institute. Deleterious PLCB4 Gene Mutation. NCI Thesaurus. Code C160888.

A change in the nucleotide sequence of the PLCB4 gene that is associated with increased risk of disease. 